\title{
INTEGRATED USE OF REMOTE SENSED DATA AND NUMERICAL CARTOGRAPHY FOR THE GENERATION OF 3D CITY MODELS
}

\author{
Gabriele Bitelli, Valentina Alena Girelli*, Alessandro Lambertini \\ DICAM, Dept. of Civil, Chemical, Environmental and Materials Engineering (DICAM), \\ Alma Mater Studiorum University of Bologna, Italy \\ (gabriele.bitelli, valentina.girelli, alessandro.lambertini)@unibo.it
}

Commission II, WG II/4

KEY WORDS: 3D City Model, Numerical cartography, SmartCity, Data fusion, SfM, Oblique imagery, LiDAR

\begin{abstract}
:
3D city models are becoming increasingly popular and important, because they constitute the base for all the visualization, planning, management operations regarding the urban infrastructure. These data are however not available in the majority of cities: in this paper, the possibility to use geospatial data of various kinds with the aim to generate 3D models in urban environment is investigated.

In 3D modelling works, the starting data are frequently the 3D point clouds, which are nowadays possible to collect by different sensors mounted on different platforms: LiDAR, imagery from satellite, airborne or unmanned aerial vehicles, mobile mapping systems that integrate several sensors. The processing of the acquired data and consequently the obtainability of models able to provide geometric accuracy and a good visual impact is limited by time, costs and logistic constraints.

Nowadays more and more innovative hardware and software solutions can offer to the municipalities and the public authorities the possibility to use available geospatial data, acquired for diverse aims, for the generation of 3D models of buildings and cities, characterized by different level of detail.

In the paper two cases of study are presented, both regarding surveys carried out in Emilia Romagna region, Italy, where 2D or 2.5D numerical maps are available. The first one is about the use of oblique aerial images realized by the Municipality for a systematic documentation of the built environment, the second concerns the use of LiDAR data acquired for other purposes; in the two tests, these data were used in conjunction with large scale numerical maps to produce 3D city models.
\end{abstract}

\section{INTRODUCTION}

Today a city to be defined "smart" must be first of all "digital”. In fact, in urban planning and management a detailed and accurate 3D city model can be used for a large amount of different requests: the visualization and analysis of the surrounding environment in the building's design, the planning of restoration interventions and new infrastructures construction, the development of communication networks, the preparation of emergency plans in case of disasters, the simulation of future situations, the modelling of some complex and important environmental parameters, as for example the heat demand, the solar radiation, the air pollution, the urban noise, the shadows estimations, the energetic requirement, etc. (Zlatanova et al., 2004; Biljecki et al., 2015; Bitelli et al., 2015).

These 3D city models can be seen as the efficient backbone to be used to collect, integrate and manage heterogeneous geospatial information. In fact, for these applications different kinds of data coming from a plethora of sources are required and, in order to permit their use with a greater level of efficiency, they must be as accurate as possible and kept always up-to-date.

These results cannot be achieved with traditional survey systems because they would require a long time to survey and process large areas such as whole cities, inevitably increasing the costs of the final 3D city model. On the other hand, many municipalities dispose of a $2 \mathrm{D}$ or $2.5 \mathrm{D}$ geometrical database. Whilst some authors have tried to derive the data for the $3 \mathrm{D}$ models starting from 2D representation and other kinds of information, coming for example from textual or heterogeneous data (Biljecki et al., 2017; Gatta et al., 2017), the availability of geospatial datasets can provide more reliable information.

For this reason, the development of automated or semiautomated methods and procedures for 3D buildings extraction and reconstruction from remotely sensed data is now crucial, providing different kinds of Level of Detail (LOD) (Kolbe et al., 2005). The latest developments in sensors, platforms and algorithms, suitable for obtaining and processing geospatial data, allow responding to this need with effective solutions.

3D city models allow an efficient bi-directional integration in a GIS with data coming from different information systems (Ross et al., 2009; Africani et al., 2013). On the other part of the scale, these models can benefit from the highest LOD achievable with information obtained from Building Information Models (BIM) (Barazzetti \& Banfi, 2017; de Laat \& van Berlo, 2011; Floros et al., 2017; Kang \& Hong, 2015; Ma \& Ren, 2017). Applications that can make effective use of the mentioned systems are many, both for mapping and updating the current representation of the territory and for monitoring changes over time.

Several survey solutions available today allow to acquire geospatial data in different contests, with different systems and different resolution; in general, it is possible to identify three macro-groups of main remote sensing platforms to be used for data acquisition: spaceborne, airborne and the so-called Unmanned Aerial Vehicle (UAV), to which must be added also the terrestrial platforms, often mobile in urban surveying applications. These platforms can mount different types of

* Corresponding author 
sensors, alone or in integration: Aerial Laser Scanners (ALS), Terrestrial Laser Scanners (TLS), aerial or terrestrial digital cameras, thermal cameras, Radio Detection and Ranging (RADAR), etc.

Each system has peculiar capabilities, characteristics, potentialities and problems and the final choice should depend by the requirements of the final products to be obtained at the end of the whole process: the surveying is in fact only the first step of a complex workflow, in which the data processing plays a crucial role. Especially in the phase of elaboration, there is the research challenge of the 3D city modelling (Biljecki et al., 2015; Zhu et al., 2009). In fact the obtainability of models able to provide the accurate geometric information needed for the uses previously described, is limited by time, costs and logistic constraints; it has thus become increasingly pressing the requirement of software and algorithms more and more directed towards robust fully automatic methods for the 3D city models. The final aim of the research in this field is the reduction of the human operator intervention, in many cases still necessary especially in the phase of interpretation, analysis, revision and editing of the results, generating higher costs and time consuming.

In this paper two cases of study and the respective survey techniques and data processing methodologies are illustrated, to stress the aforementioned procedures, demonstrating each approach's strengths and weaknesses and evidencing related potentialities and problems.

The raw starting data in the presented works are essentially point clouds derived by LIDAR or by processing aerial images within Structure from Motion (SfM) software; all the surveys have been carried out in Emilia Romagna region, in Italy.

In both cases, open data as for example the available regional and municipal digital maps and orthophotos have been used for different aims depending on the specific needs: as reference for the orientation of the aerial images, as source for the generation of the buildings 3D models, as a base on which integrating the generated 3D models, offering the possibility to create graphic products characterized by a high visual impact and supporting different GIS related applications.

\section{3D CITY MODELLING BY OBLIQUE AERIAL} IMAGES

The first case of study is about the use of oblique aerial images for 3D city modelling. The use of oblique imagery, in innovative airborne sensors and also in integration with LiDAR, is becoming a standard in the field of urban mapping for generating photo-realistic 3D models of buildings and cities; the image-based rendering is today an increasingly popular request, especially in Cultural Heritage, game and movie industry and urban planning, also because the texture creates the impression of a higher level of detail.

Using oblique images instead of nadir ones offer many benefits: buildings' roofs and facades are visible, with the same flight path it is possible to have larger images overlap and consequently higher redundancy together with lesser occlusions, the buildings' geometry interpretation is easier, the automatic generation of the true-orthophoto permits to obtain more photo-realistic products.

On the other hand, the processing of this type of images requires specific procedures, because of some critical issues: many software aren't able to manage aerial convergent images, presenting variable factor scale and geometric resolution, perspective distortions, different illumination and shadow areas.
Software using the SfM approach can partially offer an answer to these requirements, even if some challenges remain open, as for example the modelling of historical city centres with buildings characterized by unconventional shapes (Toschi et al, 2017).

In the presented work, an oblique aerial images block has been processed using two different SfM software packages; even if the comparison between the software performances isn't the primary aim of the presented work, the obtained final results are presented, qualitatively evaluated and analysed in their visual output, underlying the problems encountered during the elaboration.

\subsection{The imagery dataset}

The images have been provided by the Municipality of Bologna, which regularly perform the acquisition of oblique imagery as a support for the urban management, a powerful instrument in the documentation of the current situation and the changes occurred in time on the city buildings.

The block used in the test consists of 132 images, acquired in May 2017, and the interested area is about of $1.5 \mathrm{sq} \mathrm{km}$, with high buildings and green flat zones. This district, located North in Bologna, has been chosen because it is the subject of energetic efficiency analysis in the frame of the ChoT project (The Challenge of remote sensing Thermography as indicator of energy efficiency of buildings) of the DICAM Dept. (Mandanici \& Conte, 2016). The generated buildings' 3D model may in fact constitute another important factor in the evaluation of energy efficiency in this urban area.

The images were acquired by the CGR SPA Company (Parma, Italy) using the MIDAS 5 system, developed by Track'Air B.V. The standard system consists of four $45^{\circ}$ tilted and one nadir Canon EOS-1Ds Mk3 full frame cameras, connected to a dedicated data acquisition computer (Figure 1). The focal length is $85 \mathrm{~mm}$ in the vertical camera and $100 \mathrm{~mm}$ in the oblique ones; consequently, being the flight altitude of $1000 \mathrm{~m}$, the average images GSD is about $8 \mathrm{~cm}$.

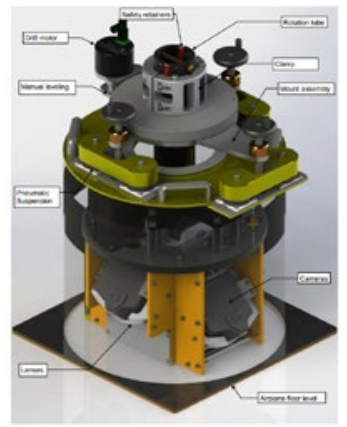

Figure 1. MIDAS 5 system assembly (www.trackair.com).

For the images orientation 15 ground control points and 10 tie points derived by the 1:2000 municipal digital map of Bologna have been used; this numerical map stores for the buildings the height at the footprint and at the eaves, but a true there dimensional textured city model is not available. The contrl points, regularly spaced in the whole area of interest, are located both at ground and eaves heights.

\subsection{Data processing}

Image processing has been performed using two different SfM software packages, Bentley ContextCapture $@$ and Agisoft 
PhotoScan Pro@

The processing phases, similar for both software, are the classic ones of SfM procedure (Figure 2). The performance of the two packages prove to be comparable, also in terms of time consuming, that results of 5/6 hours for the entire process.

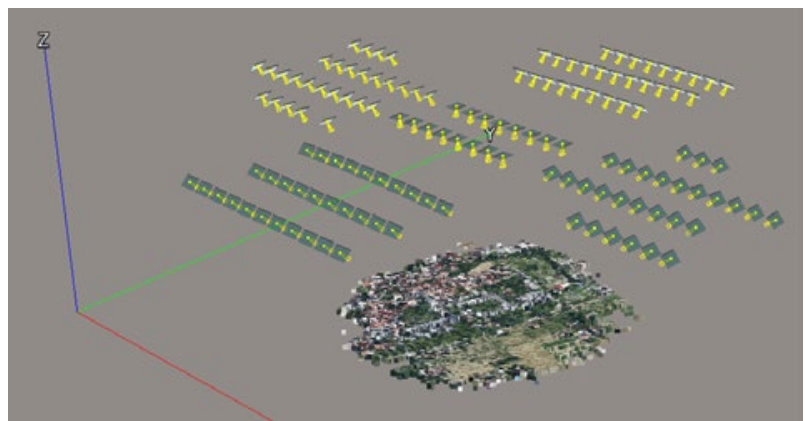

Figure 2. Images acquisition scheme and point cloud generated by ContextCapture.

Table 1 shows some values, which permit to qualitatively evaluate the processing; a more in-depth comparison is beyond the scope of this work.

As shown, ContextCapture has extracted a much more populated dense point cloud, even if the average spacing is lightly higher compared to the cloud generated by PhotoScan, that appears to be more homogeneously spaced; this fact demonstrates that ContextCapture seems to be more able to create a point cloud which adapts better to the morphology of the object, increasing the point spacing only where it is necessary.

\begin{tabular}{|c|c|c|c|}
\hline $\begin{array}{c}\text { Reproj. } \\
\text { Error (px) }\end{array}$ & $\begin{array}{c}\text { Dense Cloud } \\
\text { (N. of points) }\end{array}$ & $\begin{array}{c}\text { Average } \\
\text { points spacing }\end{array}$ & $\begin{array}{c}\text { Mesh (N. of } \\
\text { triangles) }\end{array}$ \\
\hline \multicolumn{4}{|c|}{ ContextCapture } \\
\hline 0.52 & $\approx 350$ millions & $5 \mathrm{~cm}$ & $\approx 7$ millions \\
\hline \multicolumn{4}{|c|}{ PhotoScan Pro } \\
\hline 0.67 & $\approx 87$ millions & $4 \mathrm{~cm}$ & $\approx 18$ millions \\
\hline
\end{tabular}

Table 1. Quality indicators of the images processing.

\subsection{Evaluation of the final products}

From a visual analysis the generated final products (point cloud, textured mesh and true orthophoto) have a good quality in both software, and results suitable for representation purposes. In Figure 3 is presented the true orthophotos obtained by ContextCapture with superimposed, in yellow, the layer "buildings" from the municipal digital map (Coordinate System WGS84/UTM 32N).

In Figure 4 is reported the same detail of the photo-textured 3D model generated by the two software.

This figure demonstrates that, despite a lesser total number of triangles, the model generated by ContextCapture presents a better detail, especially in buildings' facades reconstruction and in the resolution of the texture.

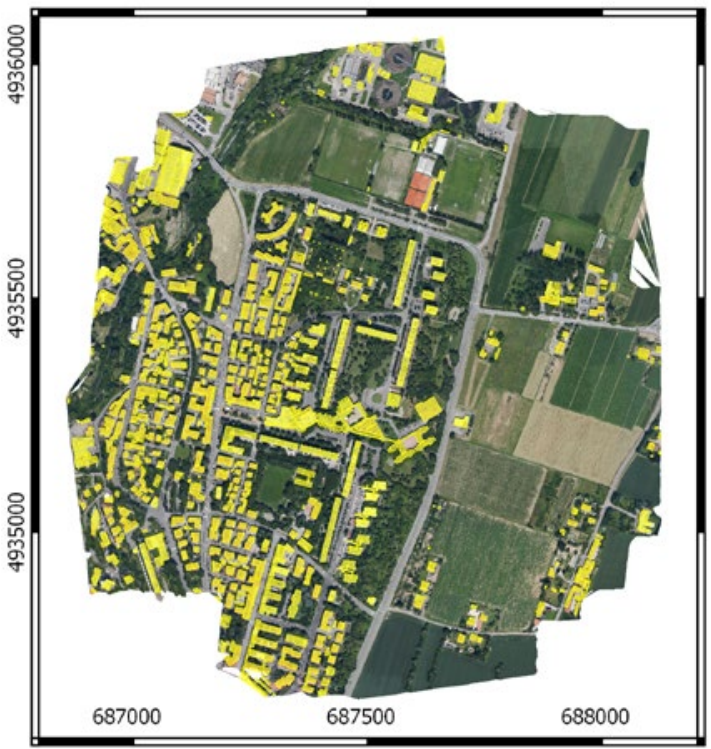

Figure 3. The true orthophoto (by ContextCapture)
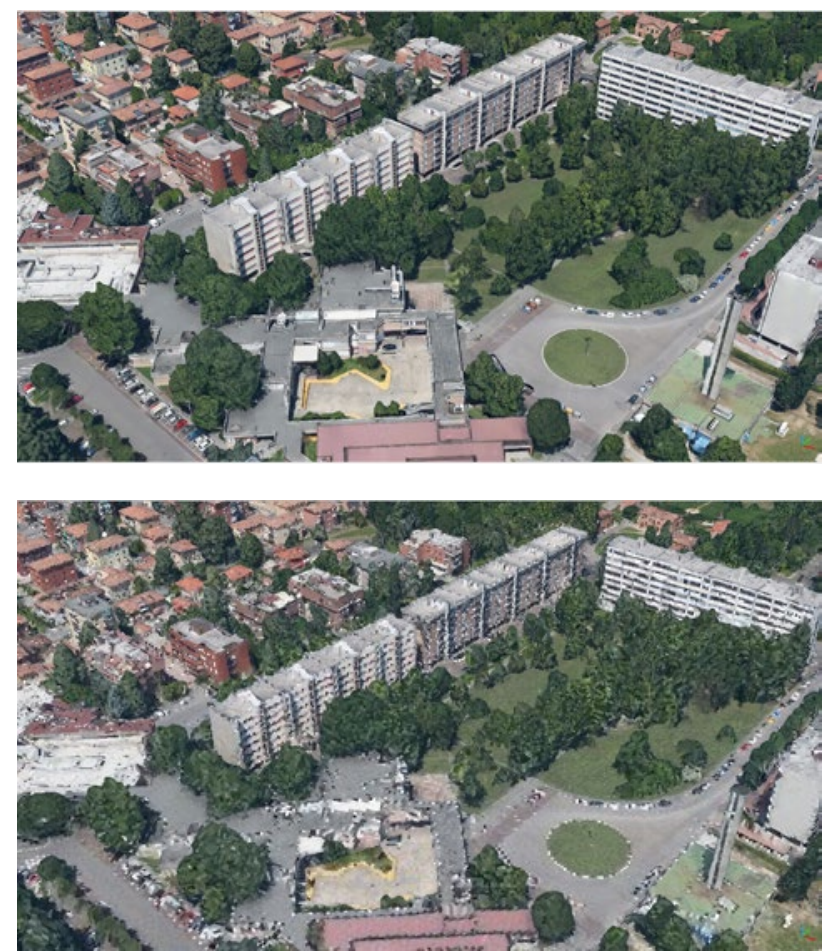

Figure 4. A detail of the obtained 3D model. Top: by ContextCapture, bottom: by PhotoScan.

The numerical comparison between the two dense point cloud shows a good agreement; in fact, the cloud-to-cloud average distance is in the order of few centimetres (excluding by the comparison the part of the vegetation areas which obviously show highest differences, in grey in the Figure 5), even if this value increases in the buildings' edges and facades, reaching values up to 1 meters in some cases (red points). 


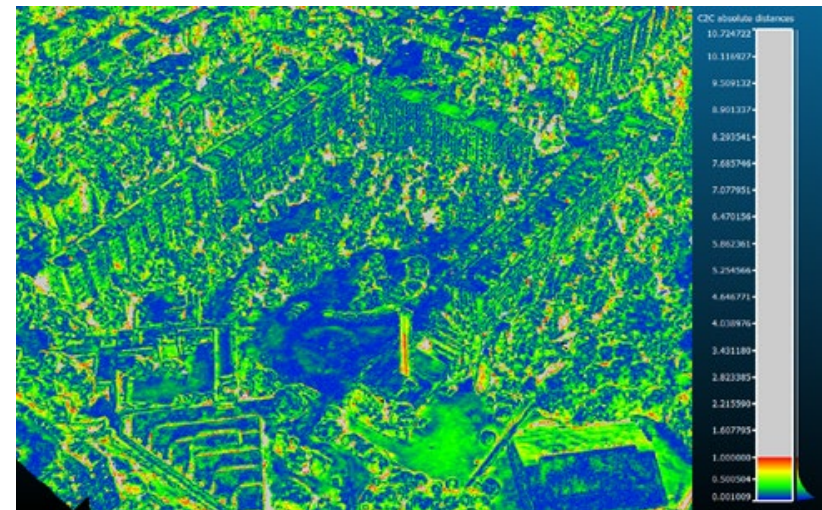

Figure 5. Result of the "cloud-to-cloud" distance computation between the dense cloud generated by ContextCapture and PhotoScan Pro.

A vertical section extracted in this area shows, according to what has been explained, that the cloud by PhotoScan is in fact a bit more noisier (Figure 6).
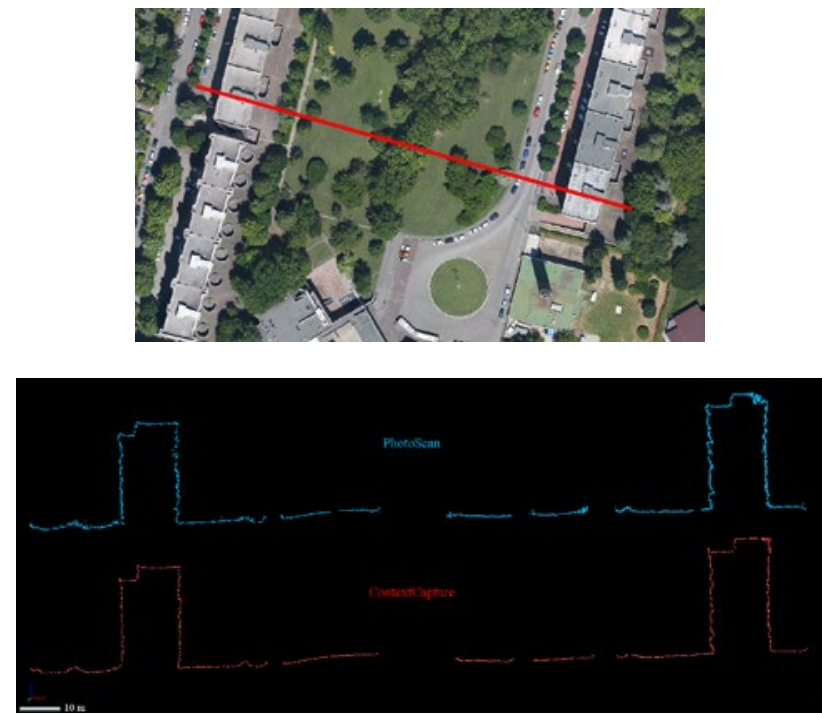

Figure 6. Section of the two point clouds. Top: the section plane, bottom: the obtained result (red: ContextCapture, blue: PhotoScan Pro).

\section{GENERATION OF A 3D CITY MODEL IN GIS ENVIRONMENT BY DIGITAL MAP AND LIDAR DATA}

The second case of study concerns the use of LiDAR data integrated and fused together with data acquired from other sensors and platforms and released as Open Data: a large scale numerical cartography of a coastal city. The conjunct use of various geomatic techniques has demonstrated to be a productive approach to deliver updates for urban databases of geospatial data (Franci et al., 2014).

Considering this particular case of study, LiDAR data was not specifically surveyed for the generation of a 3D city model. It must be mentioned that the original purpose of the ALS survey was the monitoring of coastal changes due to the erosion phenomenon, but the acquired data proved to be suitable also for building modelling following a specific procedure.

The mentioned procedure has been performed within a free and open-source GIS software (FOSS) environment; in this chapter the phases of the processing are shown, highlighting also some issues arising from the use of datasets not expressly acquired with the aim of the generation of a 3D City Model. Some critical situations, related to specific building shapes or the geometrical characteristics of data acquisition, are discussed by examples. The results show the possibility of such a hybrid approach to achieve a simplified yet powerful 3D city model with reduced costs exploiting already surveyed datasets.

The processed point cloud is a subset of the acquired LiDAR strip over the city of Riccione (Italy), which is 500 meters wide approximately centred on the coastline.

As a preliminary analysis, a DSM (Digital Surface Model) and a filtered DTM (Digital Terrain Model) is processed from the point cloud (Figure 7).

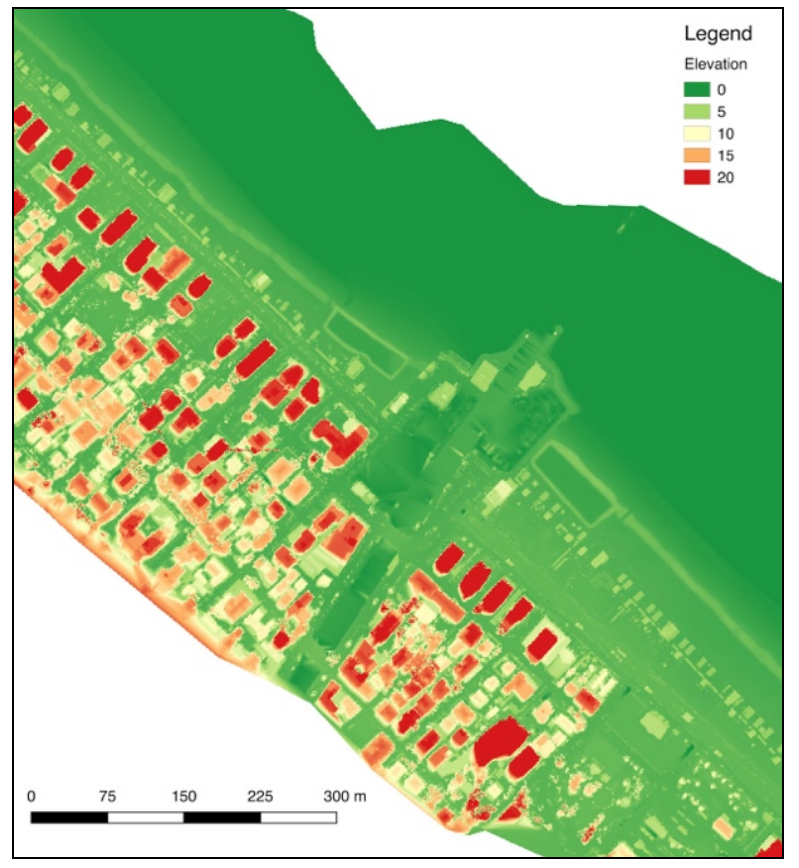

Figure 7. DSM from the processed LiDAR data.

Isolating only the points classified as "ground" within the point cloud, the DTM is computed and later used as a reference for the computation of the elevation of each building.

The aim of the procedure is to generate, in a GIS environment, a $3 \mathrm{D}$ city model using data from heterogeneous databases such as point cloud and the municipal numerical cartography, therefore extending the life and impact of geospatial data already surveyed, within the context and the good practice of public data reuse, returning back new information as output of the processing, used to create new attributes for an updated urban cartography.

Furthermore, the following workflow is a powerful solution that is also simple to replicate in other cases of study, with a high level of automation through the entire procedure; this is particularly useful for urban planning, providing a better representation of the geometrical features of each surveyed building.

According to the definition of Kolbe et al. (2005), the numerical cartography currently available for the municipality has a basic Level-of-Detail equal to LoD0, compiling with CityGML, composed by a 2.5D DTM and a geometry layer of closed polygons representing the perimeters of each building. In order to increase the LoD, this work relies on altimetry information extracted from LiDAR data, generating a new model with a LoD1, reconstructing the geometry of buildings as block models. 
Relying on high density point cloud, it would be possible to precisely produce a 3D model of each sloped roof (Africani et al., 2013), obtaining a LoD2 output with distinctive roof structures: ridges, slopes, etc. Processing the data available for our case of study, however, it is only possible to create a LoD1 output, due to the low density of the point cloud.

Nevertheless, thanks to the use of appropriate filters, the points classified as "not ground", surveyed over roof surfaces, are used to compute the elevation of each building. In the proposed procedure, each building perimeter is acquired from the numerical cartography and then used as a mask.

Some predictable issues must be discussed and considered appropriately, such as problems due to outliers, occlusions, shift between datasets or noise from data acquired over the façade (Figure 8).

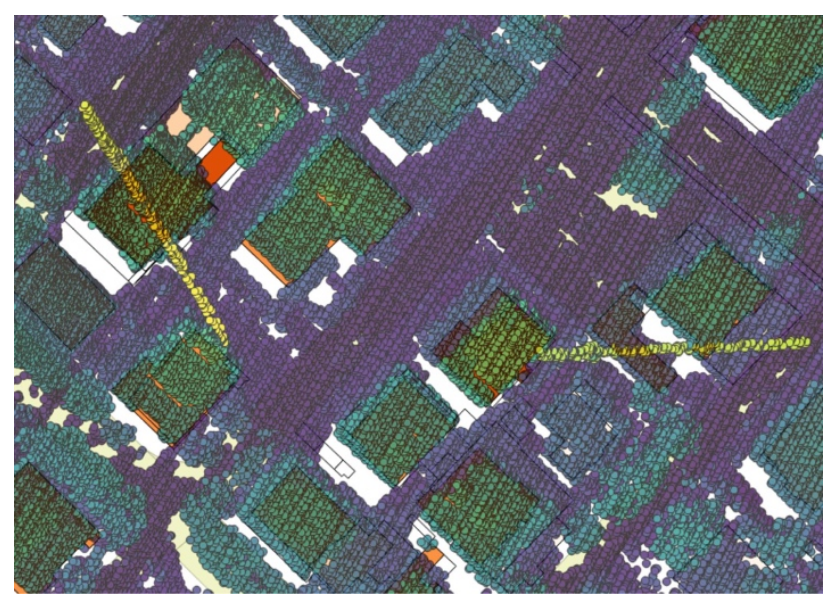

Figure 8. Example of some issues in the datasets: outliers, shift and occlusions.

As previously mentioned, the LiDAR survey was performed in order to detect coastal changes. Therefore, the flight mission was carried out following the coastal line at nadir. One of the critical issues in the reuse of the LiDAR dataset, to provide altimetry information for inland buildings, regarded the resulting slanted line of sight between the ALS sensor and the surveyed objects, i.e. the buildings. It must be considered that, due to the oblique angle of acquisition, some occlusions will inevitably result in the processing of data very close to the border of the field of view of the instrument.

Furthermore, as another consequence of the diagonal angle of the ALS, a significant number of points are also detected over each façade of the building parallel to the flight line, as an opposite effect of the aforementioned occlusions.

Additionally, a slight shift in georeferencing and alignment between the two datasets, implicit in the nominal accuracy of LiDAR and numerical cartography, could generate gross errors in derived products.

Considering all the described premises, in order to avoid any issues on the quality of the final product, a margin of tolerance should be considered over the footprint of each building, through the application of a buffer around the geometry, following a basic GIS procedure. Thanks to this precaution, both the slight misalignment and the façade's points related problems are in fact resolved.

The computation of the elevation of each building is performed at different stages: at ground level (base), at the gutter line and at the ridge of the slopes (Figure 9).

In order to produce a coherent block model, compliant with a LoD1, due to the low density of the point cloud, only an average elevation value of each roof is considered.

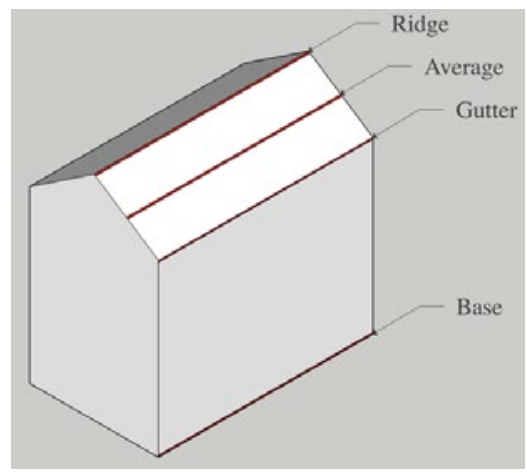

Figure 9. Elevation data for each building in the case study.

The average value is computed from the corresponding points of the LiDAR cloud, individually masked with the perimeter geometry of each building, following an iterative procedure, considering also the application of an internal buffer as previously mentioned.

The absolute elevation value, obtained from ALS data, is then normalized for each building by a simple subtraction of the DTM elevation value at the base.

Therefore, relative elevation is computed for each building and then its value is transferred back as a new attribute in an updated version of the urban numerical cartography.

In this final step, one of the possible output of the data fusion between numerical cartography and LiDAR data is produced as a thematic map that represent the computed elevation of each building (Figure 10).

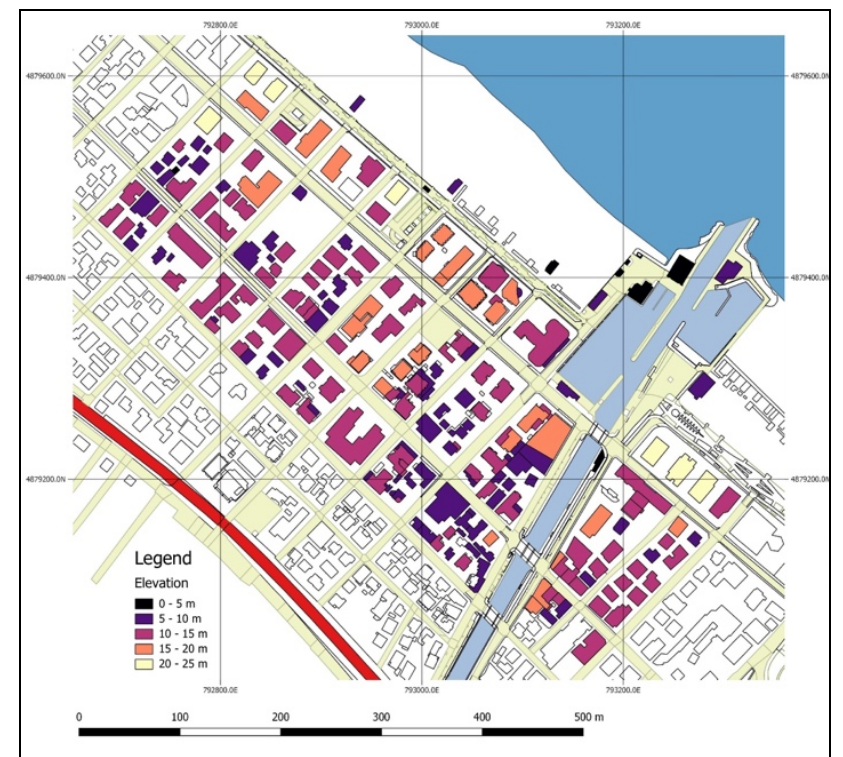

Figure 10. Output represented as a thematic map.

From the processed information, it is also simple to derive further attributes related to each building such as the volume, computed from the value of the area obtained from the numerical cartography, combined with the elevation obtained from the ALS survey.

Finally, it is also possible to rapidly produce a complete 3D City Model to graphically represent the actual elevation of each building in an interactive three-dimensional environment. These products are suitable to provide a practical interface to users and public stakeholders, in order to better understand the derived geographic information (Figure 11). 


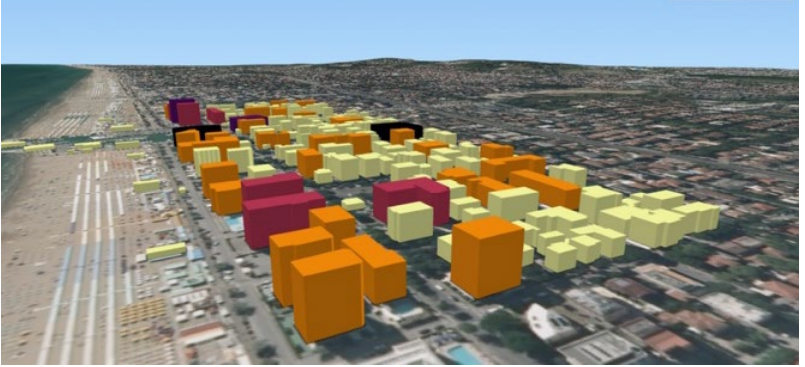

Figure 11. 3D City Model graphical representation with LoD1 buildings classified according to their new volume attribute.

The proposed workflow is completely automated and it proved to be a fast and effective processing method to rapidly deliver results. It is also easily replicable in other cases of study providing a new use of already surveyed datasets.

\section{CONCLUSIONS}

A 3D city model is today a reality of great interest in the management of the city and in support of information systems of different content and purpose. If the generation of such data is still not a widespread practice, also because of the high costs, the application of geomatic techniques has the potential to provide useful solutions even starting from digital data already available, not specifically created for the generation of 3D city models. The two examples show how this objective can be achieved with different products but with significant results for the municipalities.

\section{ACKNOWLEDGEMENTS}

Authors would like to thank the Municipality of Bologna (A. Minghetti, E. Paselli, P. Africani) and the Municipality of Riccione (O. Tomasetti, G. Ovani) for making available all the data processed in the presented work, and the company CGR $\mathrm{SpA}$, for providing information about oblique images used for the test. Many thanks to the students Erika Roncagli, Anna La Piana and Filippo Polverelli. This work has been partially supported by the project "GAMHer: Geomatics Data Acquisition and Management for Landscape and Built Heritage in a European Perspective", PRIN Progetti di Ricerca di Rilevante Interesse Nazionale - Bando 2015, Prot. 2015HJLS7E.

\section{REFERENCES}

Africani, P., Bitelli, G., Lambertini, A., Minghetti, A., Paselli, E., 2013. Integration of LiDAR data into a municipal GIS to study solar radiation. Int. Arch. Photogramm. Remote Sens. Spat. Inf. Sci., XL-1/W1, 1-6.

Barazzetti, L., Banfi, F., 2017. BIM and GIS: when parametric modeling meets geospatial data. ISPRS Annals of the Photogrammetry, Remote Sensing and Spatial Information Sciences, Vol.IV-5/W1, pp. 1-8.

Biljecki, F., Stoter, J., Ledoux, H., Zlatanova, S., Çöltekin, A., 2015. Applications of 3D City Models: State of the Art Review. ISPRS Int. J. Geo-Information 4, pp. 2842-2889. doi:10.3390/ijgi4042842.

Biljecki, F., Ledoux, H., Stoter, J. 2017. Generating 3D city models without elevation data. ISPRS Computers, Environment and Urban Systems 64, pp. 1-18.
Bitelli, G., Conte, P., Csoknyai, T., Franci, F., Girelli, V.A., Mandanici, E., 2015. Aerial thermography for energetic modelling of cities. Remote Sens. 7, pp. 2152-2170. doi:10.3390/rs70202152.

de Laat, R., van Berlo, L., 2011. Integration of BIM and GIS: The Development of the CityGML GeoBIM Extension. In: Kolbe T., König G., Nagel C. (eds) Advances in 3D GeoInformation Sciences. Lecture Notes in Geoinformation and Cartography. Springer, Berlin, Heidelberg. doi:10.1007/978-3642-12670-3_13.

Floros, G., Pispidikis, I., Dimopoulou E., 2017. Investigating integration capabilities between IFC and CityGML LOF3 for 3D City Modelling. Int. Arch. Photogramm. Remote Sens. Spat. Inf. Sci., XLII-4/W7, 1-6. doi:10.5194/isprs-archives-XLII-4W7-1-20.

Gatta, G., Arioti, E., Bitelli, G., 2017. Geomatics science applied to cartographic heritage and archive sources: A new way to explore the XIXth century Gregorian Cadastre of Bologna (Italy), an ante-litteram 3D GIS. Journal of Cultural Heritage 23, pp. 68-76.

Kang, T.W., Hong, C.H., 2015. A study on software architecture for effective BIM/GIS-based facility management data integration. Automation in Construction, 54, art. no. 1887, pp. 25-38.

Kolbe, T.H., Gröger, G., Plümer, L., 2005. CityGMLInteroperable Access to 3DCity Models. Geo-Information Disaster Manag., pp. 883-900. doi:10.1007/3-540-27468-5_63.

Franci, F., Lambertini, A., Bitelli, G., 2014. Integration of different geospatial data in urban areas: a case of study. Proc. of SPIE 9229, pp. 92290P-1 - 92290P-9.doi:10.1117/12.2066614.

Ma, Z., Ren Z., 2017. Integrated Application of BIM and GIS: An Overview. Procedia Engineering, Volume 196, pp. 10721079, ISSN 1877-7058. doi:10.1016/j.proeng.2017.08.064.

Mandanici, E., Conte, P., 2016. Aerial thermography for energy efficiency of buildings: the ChoT project. Proc. of SPIE 10008, 1000808. doi: $10.1117 / 12.2241256$

Qing Zhu, Q., Hu, M., Zhang, Y., Du, Z., 2009. Research and practice in three-dimensional city modeling, Geo-spatial Information Science, 12:1, pp. 18-24, doi:10.1007/s11806-0090195-z.

Ross, L., Bolling, J., Döllner, J., Kleinschmit, B., 2009. Enhancing 3D City Models with Heterogeneous Spatial Information: Towards 3D Land Information Systems. In M. Sester et al. (eds.), Advances in GIScience, Lecture Notes in Geoinformation and Cartography, pp. 113-133.

Toschi, I., Ramos, M.M., Nocerino, E., Menna, F., Remondino, F., Moe, K., Poli, D., Legat, D., Fassi, F., 2017. Oblique photogrammetry supporting 3D urban reconstruction of complex scenarios. Int. Arch. Photogramm. Remote Sens. Spatial Inf. Sci., XLII-1/W1, pp. 519-526, doi:10.5194/isprsarchives-XLII-1-W1-519-2017.

Zlatanova, S., Holweg, D., 2004. 3D Geo-Information in Emergency Response: A Framework. In: Proceedings of the 4th International Symposium on Mobile Mapping Technology (MMT 2004), March 29-31, Kunming, China. 